\title{
LEARNING REDUCED SYSTEMS VIA DEEP NEURAL NETWORKS WITH MEMORY
}

\author{
Xiaohan Fu, ${ }^{1, *}$ Lo-Bin Chang, ${ }^{1}$ \& Dongbin Xiu ${ }^{2}$ \\ ${ }^{1}$ Department of Statistics, Ohio State University, Columbus, OH 43210, USA \\ ${ }^{2}$ Department of Mathematics, Ohio State University, Columbus, $\mathrm{OH} 43210$, \\ USA
}

*Address all correspondence to: Xiaohan Fu, Department of Statistics, Ohio State University, Columbus, OH 43210, USA, E-mail: fu.688@osu.edu, lobinchang@stat.osu.edu,xiu.16@osu.edu

Original Manuscript Submitted: 3/20/2020; Final Draft Received: 6/23/2020

We present a general numerical approach for constructing governing equations for unknown dynamical systems when data on only a subset of the state variables are available. The unknown equations for these observed variables are thus a reduced system of the complete set of state variables. Reduced systems possess memory integrals, based on the well-known Mori-Zwanzig (MZ) formulation. Our numerical strategy to recover the reduced system starts by formulating a discrete approximation of the memory integral in the MZ formulation. The resulting unknown approximate $M Z$ equations are of finite dimensional, in the sense that a finite number of past history data are involved. We then present a deep neural network structure that directly incorporates the history terms to produce memory in the network. The approach is suitable for any practical systems with finite memory length. We then use a set of numerical examples to demonstrate the effectiveness of our method.

KEY WORDS: deep neural network, reduced system, Mori-Zwanzig formulation, memory integral

\section{INTRODUCTION}

Designing data-driven numerical methods to discover unknown physical laws has received an increasing amount of attention lately. Several methods were developed for dynamical systems by using traditional numerical approximation techniques. In these approaches, the unknown governing equations are treated as target functions, whose inputs are the state variables and whose outputs are their temporal derivatives. Methods using sparse recovery, as well as more standard polynomial approximations, have been developed (Brunton et al., 2016; Kang et al., 2019; Long et al., 2018a,b; Raissi, 2018; Raissi et al., 2017a,b; Rudy et al., 2018; Schaeffer, 2017; Schaeffer and McCalla, 2017; Schaeffer et al., 2018; Sun et al., 2019; Tibshirani, 1996; Tran and Ward, 2017; Wu et al., 2019; Wu and Xiu, 2019). Currently research is devoted to the use of modern machine learning techniques, particularly deep neural networks (DNNs). The studies include recovery of ordinary differential equations (ODEs) (Qin et al., 2019a; Raissi et al., 2018; Rudy et al., 2019) and partial differential equations (Long et al., 2018a,b; Raissi, 2018; Raissi et al., 2017a,b; Sun et al., 2019). A notable development along this line of approach is the use of flow maps for modeling the unknown dynamical equations (Qin et al., 2019a). Flow map describes 
the (unknown) mapping between two system states. Once it is accurately approximated, it can serve as a model for system prediction. The major advantage of using flow map is that it avoids requiring temporal derivative data, which can be difficult to acquire in practice and often subject to larger errors. In particular, residual network (ResNet), developed in the image analysis community (He et al., 2016), is particularly suitable for equation recovery, in the sense that it can be an exact integrator (Qin et al., 2019a). This approach has since been extended and applied to other problems (Chen and Xiu, 2020; Qin et al., 2020; Wu and Xiu, 2020).

The aforementioned approaches are data driven and rely on observational data of the state variables to numerically estimate the underlying dynamical systems. For many practical systems, however, we do not have access to data for all the state variables. Instead, we often have data on only a subset of the variables, the observables. It is then natural to seek a governing equation for the evolution of the observed variables. This, however, introduces additional challenges from a mathematical point of view. Even when the underlying governing equations for the full variable set are autonomous, the effective governing equations for the observed variables (i.e., the reduced system of equations) include memory terms and become nonautonomous. This is a direct result of the well-known Mori-Zwanzig (MZ) formulation (Mori, 1965; Zwanzig, 1973). The memory term in the MZ formulation represents a significant computational challenge. Various approximation techniques have been developed to facilitate efficient estimation of the memory effect. See, for example, Bernstein (2007), Chertock et al. (2016), Chorin et al. (2002), Hald and Stinis (2007), Stinis (2007), Venturi and Karniadakis (2014), Zhu and Venturi (2018), and the references therein. And more recently, data-driven methods were developed to provide effective closure or estimation for memory integrals (Brennan and Venturi, 2018; Lei et al., 2016).

The topic of this paper is on data-driven learning of unknown dynamical systems when only data on a subset of the state variables (i.e., observables) are available. We make a general assumption that the underlying unknown system of complete equations are autonomous. Our goal is to construct a dynamical model for the evolution of the observables, whose data are available, thus discovering a reduced system. Due to the MZ formulation, the unknown governing equations for the observables are non-autonomous and possess memory integrals. Therefore, the aforementoned existing data-driven methods for equation discovery are not applicable. On the other hand, the existing approximation techniques for the memory integral in $\mathrm{MZ}$ equations are not applicable either, as the underlying complete system is unavailable. We therefore propose a new method to directly learn the evolution equations for the observables, with a built-in memory effect. To accomplish this, we make a general assumption that the reduced systems for the observables have "decaying memory" over a longer time horizon. When the observables are representative of the full system states, this usually holds true as the evolution of the observables depends on their current states and their immediate past, and the dependence does not usually extend to infinite past. In other words, the initial states of the observables should have diminishing effects on their evolution over longer time. Based on the decaying memory assumption, we then truncate the memory integral in the MZ formulation up to its "memory length" to obtain an approximate MZ (AMZ) equation. The AMZ equation is then discretized by using a set of time instances inside the memory interval. The resulting discrete AMZ (d-AMZ) equation, still unknown at this stage, becomes our goal of equation learning. We then design a DNN structure that explicitly incorporates the observable data inside the memory interval. The proposed DNN structure is an extension of the ResNet structure used for autonomous system learning (Qin et al., 2019a). By incorporating data from immediate past, the new DNN can explicitly model the memory terms in the $\mathrm{MZ}$ equation. We remark that our current method is similar to a recent and 
independent work (Wang et al., 2020), where similar truncation and discretization of MZ formulation was proposed. However, the work of Wang et al. (2020) utilizes long short-term memory (LSTM) neural network (NN) structure to achieve the memory effect. Our proposed DNN structure takes a much simpler form, in the sense that it is basically a standard full-connected network and does not requires any "gates" as in LSTM. The new network also allows direct conceptual and numerical connection with the "true" memory of the underlying reduced systems.

This paper is organized as follows. After the problem setup in Section 2, we present the main method in Section 3. The decaying memory assumption is first discussed in Section 3.1, followed by the d-AMZ formulation in Section 3.2. The DNN structure is then presented in Section 3.3, along with its data set construction and training in Section 3.4. Numerical examples are then presented in Section 4 to demonstrate the properties of the proposed approach.

\section{SETUP AND PRELIMINARIES}

Let us consider a system of ODEs,

$$
\frac{d \mathbf{x}}{d t}=\mathbf{f}(\mathbf{x}), \quad \mathbf{x}(0)=\mathbf{x}_{0},
$$

where $\mathbf{x} \in \mathbb{R}^{n}$ are the state variables. We assume that the form of the governing equations, which manisfests itself via $\mathbf{f}: \mathbb{R}^{n} \rightarrow \mathbb{R}^{n}$, is unknown. Let $\mathbf{x}=(\mathbf{z} ; \mathbf{w})$, where $\mathbf{z} \in \mathbb{R}^{d}$ is the subset of the state variables with available data, and $\mathbf{w} \in \mathbb{R}^{n-d}$ is the unobserved subset of the state variables. Our goal is to construct an effective governing equation for the observed variables $\mathbf{z}$.

\subsection{Data}

We assume trajectory data are available only for the observables $\mathbf{z}$ and not for the full set of the state variables $\mathbf{x}$. Let $N_{T}$ be the total number of such partially observed trajectories. For each $i$-th trajectory, we have

$$
\mathbf{Z}^{(i)}=\left\{\mathbf{z}\left(t_{k}^{(i)}\right)\right\}, \quad k=1, \ldots, K^{(i)}, \quad i=1, \ldots, N_{T},
$$

where $\left\{t_{k}^{(i)}\right\}$ are discrete time instances at which the data are available, and $K^{(i)}$ is the total number of data in the $i$-th trajectory. Note that each trajectory is originated from an unknown initial condition $\mathbf{x}_{0}^{(i)}=\left(\mathbf{z}_{0}^{(i)} ; \mathbf{w}_{0}^{(i)}\right)$. For notational convenience, we shall assume a constance time step

$$
\Delta \equiv t_{k+1}^{(i)}-t_{k}^{(i)}, \quad \forall k=1, \ldots, K^{(i)}-1, \quad i=1, \ldots, N_{T} .
$$

We then seek to develop a numerical model for the evolution dynamics of $\mathbf{z}(t)$, without data on $\mathbf{w}$ and the knowledge of the full model [Eq. (1)].

\subsection{Learning of Full System}

When data on the full set of state variables $\mathbf{x}$ are available, the task of recovering the full model [Eq. (1)] is relatively more straightforward. A number of different approaches exist. In this paper, we adopt and modify the approach developed in Qin et al. (2019a), which seeks to recover the underlying flow map of Eq. (1) as opposed to the right-hand-side of Eq. (1). In particular, suppose data of the full set of state variables are available as

$$
\mathbf{X}^{(i)}=\left\{\mathbf{x}\left(t_{k}^{(i)}\right)\right\}, \quad k=1, \ldots, K^{(i)}, \quad i=1, \ldots, N_{T},
$$

Volume 1, Issue 2, 2020 
for a total number of $N_{T}$ trajectories over time instances $\left\{t_{k}^{(i)}\right\}$ with a constant step size, $\Delta=$ $t_{k+1}^{(i)}-t_{k}^{(i)}$ for all $i$ and $k$. We can then re-group the data into pairs of two adjacent time instances, for each $i=1, \ldots, N_{T}$,

$$
\left\{\mathbf{x}\left(t_{k}^{(i)}\right), \mathbf{x}\left(t_{k+1}^{(i)}\right)\right\}, \quad k=1, \ldots, K^{(i)}-1, \quad i=1, \ldots, N_{T} .
$$

Note that for autonomous system [Eq. (1)], time $t$ can be arbitrarily shifted and only the relative time difference is relevant. We can then define the data set as

$$
\left\{\mathbf{x}_{j}(0), \mathbf{x}_{j}(\Delta)\right\}, \quad j=1, \ldots, J
$$

where the total number of data pairs $J=\left(K^{(1)}-1\right)+\cdots+\left(K^{\left(N_{T}\right)}-1\right)$.

On the other hand, the autonomous full system [Eq. (1)] defines a flow map $\Phi: \mathbb{R}^{n} \rightarrow \mathbb{R}^{n}$, such that $\mathbf{x}\left(s_{1}\right)=\mathbf{\Phi}_{s_{1}-s_{0}}\left(\mathbf{x}\left(s_{0}\right)\right)$. We then have

$$
\begin{aligned}
\mathbf{x}(\Delta) & =\mathbf{x}(0)+\int_{0}^{\Delta} \mathbf{f}(\mathbf{x}(s)) d s \\
& =\mathbf{x}(0)+\int_{0}^{\Delta} \mathbf{f}\left(\boldsymbol{\Phi}_{s}(\mathbf{x}(0))\right) d s \\
& =\left[\mathbf{I}_{n}+\boldsymbol{\Psi}(\cdot)\right](\mathbf{x}(0)),
\end{aligned}
$$

where $\mathbf{I}_{n}$ is the identity matrix of size $n \times n$, and for any $\mathbf{x} \in \mathbb{R}^{n}$,

$$
\boldsymbol{\Psi}(\mathbf{x})=\int_{0}^{\Delta} \mathbf{f}\left(\boldsymbol{\Phi}_{s}(\mathbf{x})\right) d s
$$

Based on Eq. (5), it was then proposed in Qin et al. (2019b) to use ResNet (He et al., 2016) to recover the system. The ResNet has a structure of

$$
\mathbf{y}^{\text {out }}=\left[\mathbf{I}_{n}+\mathbf{N}\right]\left(\mathbf{y}^{\text {in }}\right)
$$

where $\mathbf{N}: \mathbb{R}^{n} \rightarrow \mathbb{R}^{n}$ is the operator corresponding to a fully connected DNN. Upon using the data set [Eq. (4)], the ResNet [Eq. (6)] can be trained to approximate the dynamics [Eq. (5)], with the deep network operator $\mathbf{N} \approx \Psi$.

\subsection{Mori-Zwanzig Formulation for Reduced System}

The approach in the previous section, along with most other existing equation recovery methods, does not apply to the problem considered in this paper. The reason is because here we seek to develop/discover the dynamic equations for only the observables $\mathbf{z}$, which belong to a subset of the full set of variables $\mathrm{x}$. Even though the full system [Eq. (1)] is autonomous, a crucial property required in most of the existing equation recovery methods, the evolution equations for the subset variables $\mathbf{z}$ become non-autonomous. This is well understood from the MZ formulation (Mori, 1965; Zwanzig, 1973). The evolution of the reduced set of variables $\mathbf{z}$ follows the generalized Langevin equation in the following form:

$$
\frac{d}{d t} \mathbf{z}(t)=\mathbf{R}(\mathbf{z}(t))+\int_{0}^{t} \mathbf{K}(\mathbf{z}(t-s), s) d s+\mathbf{F}\left(t, \mathbf{x}_{0}\right)
$$


The first term $\mathbf{R}$ depends only on the reduced variables $\mathbf{z}$ at the current time and is Markovian. The second term, known as the memory, depends on the reduced variables $\mathbf{z}$ at all times, from the intial time $s=0$ to the current time $s=t$. Its integrand involves $\mathbf{K}$, commonly known as the memory kernel. The last term is called orthogonal dynamics, which depends on the unknown initial condition of the entire variable $\mathbf{x}(0)$ and is treated as noise. Note that this formulation is an exact representation of the dynamics of the observed variables $\mathbf{z}$. The presence of the memory term makes the system non autonomous and induces computational challenges. We remark again that, even though various techniques exist to estimate the memory intergal (Bernstein, 2007; Chertock et al., 2016; Chorin et al., 2002; Hald and Stinis, 2007; Stinis, 2007; Venturi and Karniadakis, 2014; Zhu and Venturi, 2018), they rely on the knownledge of the full system [Eq. (1)] and are thus not applicable in the setting of this paper, where the full system is unknown.

\section{MAIN METHOD}

In this section, we discuss the details of our proposed numerical method. We first present finite memory approximation to the exact MZ formulation [Eq. (7)]. We then discuss discrete approximation to the finite memory $\mathrm{MZ}$ formulation. Our NN model is then constructed to approximate the unknown d-AMZ formulation.

\subsection{Finite Memory Approximation}

We make a basic assumption in the memory term of the MZ formulation [Eq. (7)]. That is, we assume the memory kernel $\mathbf{K}$ in the memory term decays over time; that is for sufficiently large $t>0$,

$$
|\mathbf{K}(\mathbf{z}(t-s), s)| \searrow, \quad \text { as } s \nearrow .
$$

More specifically, this is defined as follows.

Definition 3.1 (Decaying memory). A reduced system [Eq. (7)] is said to have uniformly decaying memory if, for any $\epsilon>0$, there exists a nonnegative constant $T_{M}$, which depends on $\epsilon$ but not on $t$, such that for all $t \geq T_{M}$,

$$
\left|\int_{0}^{t} \mathbf{K}(\mathbf{z}(t-s), s) d s-\int_{0}^{T_{M}} \mathbf{K}(\mathbf{z}(t-s), s) d s\right| \leq \epsilon .
$$

For any small $\epsilon$, the decaying memory assumption implies that the memory term in Eq. (7) depends only on the reduced variables $\mathbf{z}$ from its current state at time $s=t$ to its recent past up to $s=t-T_{M}$. The memory effect from the "earlier time" $s \in\left[0, t-T_{M}\right)$ is negligible, up to the choice of $\epsilon$. We remark that this assumption holds true for many practical physical systems, whose states are usually dependent upon their immediate past and do not extend indefinitely backward in time. In other words, the initial conditions have diminishing influence on the system states as time evolves. The constant $T_{M}$ is independent of $t$ and is called memory length. Its value is obviously problem dependent. Note that at the early stage of the system evolution when time $t$ is small, $t<T_{M}$, the condition [Eq. (9)] is trivially satisfied by letting $T_{M} \leftarrow \min \left(t, T_{M}\right)$ (or, by setting $\mathbf{z}(t)=0$, for $t<0$ ). Therefore, throughout the rest of the paper we shall use $T_{M}$ without explicitly stating this trivial case.

Upon adopting the decaying memory assumption [Eq. (9)], we define the following AMZ dynamical system:

$$
\frac{d}{d t} \hat{\mathbf{z}}(t)=\mathbf{R}(\hat{\mathbf{z}}(t))+\int_{0}^{T_{M}} \mathbf{K}(\hat{\mathbf{z}}(t-s), s) d s
$$

Volume 1, Issue 2, 2020 
where $T_{M}$ is the memory length for a chosen small error threshold $\epsilon$ from Eq. (9), and the noise term $\mathbf{F}$ in Eq. (7) is embedded in the approximation.

\subsection{Discrete Finite-Memory Approximation}

We now consider discrete representation of the AMZ system [Eq. (10)]. Let $\hat{\mathbf{z}}_{n}=\hat{\mathbf{z}}\left(t_{n}\right)$ be the solution at time $t_{n}=n \Delta$ over a constant time step $\Delta$. Let $T_{M}>0$ be the memory length in the AMZ equation [Eq. (10)]. Let $n_{M} \geq 1$ be the number of time levels inside the memory range of time $t_{n}$, for $n_{M}=\left\lfloor T_{M} / \Delta\right\rfloor$ such that

$$
\left\{t_{n-n_{M}}, \ldots, t_{n-1}\right\} \subset\left[t_{n}-T_{M}, t_{n}\right), \quad t_{n-n_{M}-1} \notin\left[t_{n}-T_{M}, t_{n}\right) .
$$

Note that we do not count the "current" time level $t_{n}$ as part of the memory. We then assume that there exists a finite dimensional function $\mathbf{M}$ such that, for any $t_{n}$,

$$
\left|\mathbf{M}\left(\hat{\mathbf{z}}_{n-n_{M}}, \ldots, \hat{\mathbf{z}}_{n-1}, \hat{\mathbf{z}}_{n}\right)-\int_{0}^{T_{M}} \mathbf{K}\left(\hat{\mathbf{z}}\left(t_{n}-s\right), s\right) d s\right| \leq \eta\left(t_{n} ; T_{M}, n_{M}\right),
$$

where $\eta \geq 0$ is the error. That is, we have assumed that the finite memory integral in AMZ [Eq. (10)] can be approximated by the $\left(n_{M}+1\right)$ dimensional function $\mathbf{M}$. Note that this merely assumes a finite integral can be approximated by using the values of its integrand at a set of discrete locations inside the integraton domain. This is a very mild assumption used in the theory of numerical integration, which is a classic numerical analysis topic. For example, we can always choose $\mathbf{M}$ to be a certain numerical quadrature rule using the nodes $\left\{\hat{\mathbf{z}}_{n-n_{M}}, \ldots, \hat{\mathbf{z}}_{n-1}, \hat{\mathbf{z}}_{n}\right\}$ and with established error behavior. Our approach in this paper, however, does not use any preselected approximation rule for the memory integral. Instead, we shall leave $\mathbf{M}$ unspecified and treat it as unknown.

With the discrete approximation to the memory integral in place, we now define a d-AMZ equation,

$$
\left.\frac{d}{d t} \widetilde{\mathbf{z}}(t)\right|_{t=t_{n}}=\left.\mathbf{R}(\widetilde{\mathbf{z}}(t))\right|_{t=t_{n}}+\mathbf{M}\left(\widetilde{\mathbf{z}}_{n-n_{M}}, \ldots, \widetilde{\mathbf{z}}_{n-1}, \widetilde{\mathbf{z}}_{n}\right),
$$

where $\mathbf{M}$ is defined in Eq. (11). Obviously, this is an approximation to the AMZ [Eq. (10)] at time level $t=t_{n}$, where the approximation error stems from the use of Eq. (11). Note that both $\mathbf{R}$ and $\mathbf{M}$ on the right-hand-side are unknown at this stage.

\subsection{Neural Network Structure}

The d-AMZ formulation [Eq. (12)] serves as our foundation for learning the dynamics of the reduced variables $\mathbf{z}$. It indicates that the evolution of $\mathbf{z}$ at any time $t_{n}$ depends on not only its current state $\mathbf{z}_{n}$ but also a finite $n_{M}$ number of its past history states $\left\{\mathbf{z}_{n-1}, \ldots, \mathbf{z}_{n-n_{M}}\right\}$, where the number $n_{M}$ depends on the memory length $T_{M}$ and the time step size $\Delta$. Based on this, we propose to build a DNN structure to create a mapping from $\mathbf{z}_{n}, \mathbf{z}_{n-1}, \ldots, \mathbf{z}_{n-n_{M}}$ to $\mathbf{z}_{n+1}$ and utilize the observational data on $\mathbf{z}$ to train the network.

Let us define

$$
\begin{gathered}
D=d \times\left(n_{M}+1\right) \\
\mathbf{Z}=\left(\mathbf{z}_{n}^{\top}, \mathbf{z}_{n-1}^{\top}, \ldots, \mathbf{z}_{n-n_{M}}^{\top}\right)^{\top} \in \mathbb{R}^{D},
\end{gathered}
$$


and a $(d \times D)$ matrix

$$
\widehat{\mathbf{I}}=\left[\mathbf{I}_{d}, 0, \ldots, 0\right],
$$

where a $(d \times d)$ size identity matrix $\mathbf{I}_{d}$ is concatenated by $n_{M}$ zero matrices of size $(d \times d)$. Let

$$
\mathbf{N}(\cdot ; \Theta): \mathbb{R}^{D} \rightarrow \mathbb{R}^{d}
$$

be the operator of a fully connected feedforward NN with parameter set $\Theta$. We then define a DNN in the following manner:

$$
\mathbf{z}^{\text {out }}=[\widehat{\mathbf{I}}+\mathbf{N}]\left(\mathbf{Z}^{\text {in }}\right)
$$

An illustration of this network with $n_{M}=2$ memory terms is shown in Fig. 1. It is straightforward to see that this network creates a mapping

$$
\mathbf{z}_{n+1}=\mathbf{z}_{n}+\mathbf{N}\left(\mathbf{z}_{n}, \mathbf{z}_{n-1}, \ldots, \mathbf{z}_{n-n_{M}} ; \Theta\right), \quad n \geq n_{M}
$$

\subsection{Data, Network Training and Prediction}

To train the network [Eq. (16)], we re-organize the data set [Eq. (2)]. Let $T_{M}$ be the memory length. With time step $\Delta$ chosen, we have $n_{M}=\left\lfloor T_{M} / \Delta\right\rfloor$.

For the trajectory data [Eq. (2)] on $\mathbf{z}$, let us consider each of the $i$-th trajectory data, where $i=1, \ldots, N_{T}$. We assume $K^{(i)} \geq n_{M}+2$. That is, each trajectory needs to contain at least $\left(n_{M}+2\right)$ data entries (otherwise this trajectory is discarded). We then select a sequence of $\left(n_{M}+2\right)$ data entries of consecutive time instances along this trajectory and group them into two vectors, with the first one as the concatenation of the first $\left(n_{M}+1\right)$ entries in the form of Eq. (14) and the second one as the last entry, in the following form:

$$
\left\{\mathbf{Z}_{j}^{(i)}, \mathbf{z}_{j}^{(i)}\right\}, \quad j=1, \ldots, J^{(i)}
$$

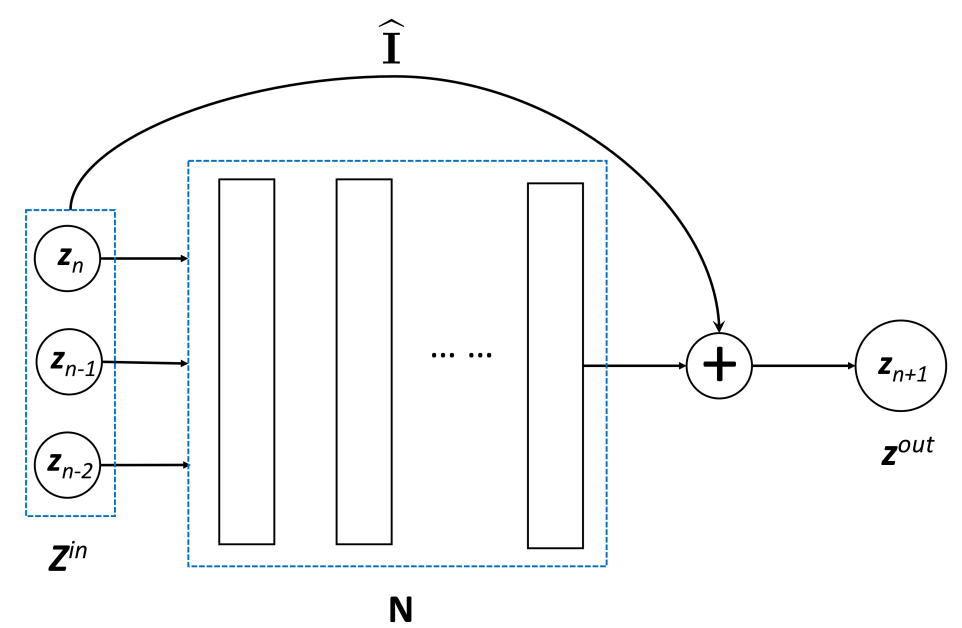

FIG. 1: Illustration of the proposed neural network with $n_{M}=2$ memory steps

Volume 1, Issue 2, 2020 
where $J^{(i)}$ is the number of such selected sequences of length $\left(n_{M}+2\right)$, and

$$
\begin{aligned}
& \mathbf{z}_{j}^{(i)}=\left[\mathbf{z}\left(t_{k_{j}}^{(i)}\right)^{\top}, \ldots, \mathbf{z}\left(t_{k_{j}+n_{M}}^{(i)}\right)^{\top}, \mathbf{z}\left(t_{k_{j}+n_{M}+1}^{(i)}\right)^{\top}\right]^{\top} \in \mathbb{R}^{D}, \\
& \mathbf{z}_{j}^{(i)}=\mathbf{z}\left(t_{k_{j}+n_{M}+2}^{(i)}\right) \in \mathbb{R}^{d}
\end{aligned}
$$

Here $k_{j}$ is the "starting" position of this sequence in the $i$-th trajectory. Obviously, when the total number of data entries along the trajectory is exactly $\left(n_{M}+2\right)$, the starting position has to be $k_{j}=1$ and the number of such groupings is $J^{(i)}=1$.

When the number of data entries is more than $\left(n_{M}+2\right)$, we may choose $J^{(i)}>1$ number of such groups. We here discuss two straightforward selections.

- Deterministic selection. This is done by selecting the starting position sequentially from $k_{j}=1$ to $k_{j}=K^{(i)}-n_{M}-1$, and then for each selected starting position take a sequence of $\left(n_{M}+2\right)$ to form the group [Eq. (18)]. This results in $J^{(i)}=K^{(i)}-n_{M}-1$ number of groups.

- Random selection. Choose $J^{(i)}<K^{(i)}-n_{M}-1$ as the number of selected groups. Randomly select $J^{(i)}$ starting position from the index set $\left\{1, \cdots, K^{(i)}-n_{M}-1\right\}$, and for each selected starting position, form a group in the form of Eq. (18).

In our numerical studies, we have found the random selection to be more effective than the deterministic selection.

The aforementioned group selection procedure is then repeated for each $i=1, \ldots, N_{T}$, trajectory. We then form the training data set by collecting all the groups from Eq. (18):

$$
\mathcal{Z}=\left\{\mathbf{Z}_{j}, \mathbf{z}_{j}\right\}, \quad j=1, \ldots, J
$$

where $J=J^{(1)}+\cdots+J^{\left(N_{T}\right)}$ is the total number of data groupings. This is the training data set, where we have re-labeled the entries using a single index $j$ for computational convenience.

By using the data set [Eq. (20)] and our network structure [Eq. (16)], we then train the NN by finding its parameter set $\Theta^{*}$ that minimizes the mean-squared loss:

$$
\Theta^{*}=\underset{\Theta}{\operatorname{argmin}} \frac{1}{J} \sum_{j=1}^{J}\left\|\mathbf{z}_{j}^{\text {out }}(\Theta)-\mathbf{z}_{j}\right\|^{2}
$$

where

$$
\mathbf{z}_{j}^{\text {out }}(\Theta)=[\widehat{\mathbf{I}}+\mathbf{N}(\cdot, \Theta)]\left(\mathbf{Z}_{j}\right), \quad j=1, \ldots, J
$$

is the network output via Eq. (16) for input $\mathbf{Z}_{j}$. Upon finding the optimal network parameter $\Theta^{*}$, we obtain a trained network model

$$
\mathbf{z}^{\text {out }}=\left[\widehat{\mathbf{I}}+\mathbf{N}\left(\cdot, \Theta^{*}\right)\right]\left(\mathbf{Z}^{\text {in }}\right) .
$$

This in turn defines a predictive model for the unknown dynamical system for the observed variables $\mathbf{z}$,

$$
\left\{\begin{array}{l}
\mathbf{z}_{n+1}=\mathbf{z}_{n}+\mathbf{N}\left(\mathbf{z}_{n}, \mathbf{z}_{n-1}, \ldots, \mathbf{z}_{n-n_{M}} ; \Theta^{*}\right), \quad n \geq n_{M} \\
\mathbf{z}_{n}=\mathbf{z}\left(t_{n}\right), \quad n=0, \ldots, n_{M}-1
\end{array}\right.
$$


With $n_{M}$ initial data on $\mathbf{z}$, we can iteratively apply the network model to predict the evolution of $\mathbf{z}$ at later time.

It is worthwhile to discuss the difference between the trained network model [Eq. (23)] and Euler forward approximation of the d-AMZ equation [Eq. (12)]. If the operators $\mathbf{R}$ and $\mathbf{M}$ in Eq. (12) are known, its Euler forward approximation takes the following form:

$$
\left\{\begin{array}{l}
\widetilde{\mathbf{z}}_{n+1}=\widetilde{\mathbf{z}}_{n}+\Delta\left[\mathbf{R}\left(\widetilde{\mathbf{z}}_{n}\right)+\mathbf{M}\left(\widetilde{\mathbf{z}}_{n}, \widetilde{\mathbf{z}}_{n-1}, \ldots, \widetilde{\mathbf{z}}_{n-n_{M}}\right)\right], \quad n \geq n_{M}, \\
\widetilde{\mathbf{z}}_{n}=\mathbf{z}\left(t_{n}\right), \quad n=0, \ldots, n_{M} .
\end{array}\right.
$$

This obviously induces temporal discretization error. In this case of Euler forward the error is $O(\Delta)$.

Although our model [Eq. (23)] and the Euler approximation [Eq. (24)] resemble each other, we emphasize that they are fundamentally different. Our NN model [Eq. (23)] is a direct nonlinear approximation to the time average of the right-hand-side of Eq. (12), whereas the Euler method [Eq. (24)] is a preselected piecewise constant approximation to the time average. Therefore, the Euler method requires the knowledge of the operators $\mathbf{R}$ and $\mathbf{M}$, which is not available in our setting, and has $O(\Delta)$ temporal error. Our NN model [Eq. (23)], on the other hand, does not contain this temporal error and uses data to directly approximate the operators in Eq. (12). For autonomous dynamical systems, it was shown that the NN model is exact in temporal integration (Qin et al., 2019b), with the only source of errors being the training error [Eq. (21)]. Error analysis for the reduced system model [Eq. (23)] in this paper is considerably more complicated, and will be pursued in separate studies.

\section{NUMERICAL EXAMPLES}

In this section, we present numerical examples to examine the performance of the proposed learning method. Our examples include two linear systems, where the exact MZ formulation for the reduced system is available; and two nonlinear systems, one of which is chaotic. Since in all examples the true models are available, we are able to compute their solutions with a high-resolution numerical solver. This creates reference solutions, with which we compare the predictive results by our NN models. For the chaotic system, an analytically defined reduced model is also available, and its results are used to compare against those of our trained reduced network model.

The training data for the reduced variables are synthetic and generated by solving the true systems with high resolution. In each example, we first choose a range of interest for the full variables $\mathbf{x}=(\mathbf{z} ; \mathbf{w}) \in D_{\mathbf{x}}$. This will be the range in which we seek an accurate model for the observed variable $\mathbf{z} \in D_{\mathbf{z}} \subset D_{\mathbf{x}}$. We then randomly generate $N_{T}$ number of initial conditions using the uniform distribution on $D_{\mathbf{x}}$. For each $i=1, \ldots, N_{T}$, we solve the underlying true system of equations with high resolution and march forward in time with time step $\Delta$. In all examples, we set $\Delta=0.02$. Each trajectory is marched forward in time for $K^{(i)}=K=$ const steps. We then only keep the trajectory data for the observed variables $\mathbf{z}$ to create our raw data set [Eq. (2)]. For benchmarking purpose, we did not add additional noises to the data. This allows us to examine more closely the properties of the method.

The memory length $T_{M}$ [Eq. (9)] is problem dependent. In each example, we progressively increase the memory length to achieve converged results. The number of memory steps is then determined as $n_{M}=\left\lfloor T_{M} / \Delta\right\rfloor$. We then randomly select $J^{(i)}$ number of sequences of length $\left(n_{M}+2\right)$ data entries from each of the $i$-th trajectory data, where $i=1, \ldots, N_{T}$, as described

Volume 1, Issue 2, 2020 
in Section 3.4, to form the training data set [Eq. (20)]. We fix $J^{(i)}=J_{0}$ to be a constant for all trajectories. The total number of data entries in the training data set [Eq. (20)] is then $J=$ $J_{0} \times N_{T}$. In all the tests, we purposefully keep the number of data $J$ to be roughly $5 \sim 10$ times of the number of parameters in the NN structure. This is to avoid any potential training accuracy loss due to lack of data and/or overfitting, thus allowing us to focus on the properties of the numerical methods. In practical computations when data are limited, proper care needs to be taken during network trianing. This is a well-recognized and well-studied topics outside the scope of this paper.

Unless otherwise noted, the NNs used in the examples consisted of three hidden layers, each of which with 30 neurons. We have used the rectified linear unit (ReLU) activation function in all the examples.

\subsection{Example 1: Small Linear System}

We first consider a simple linear system

$$
\left\{\begin{array}{l}
\dot{x}_{1}=x_{1}-4 x_{2}, \\
\dot{x}_{2}=4 x_{1}-\alpha x_{2},
\end{array}\right.
$$

where $\alpha$ is a parameter controlling the decay rate of the solution. We set $z=x_{1}$ to be the observed variable and $w=x_{2}$ to be the unobserved variable. Note that this is a simplified case of the well-documented case

$$
\left\{\begin{array}{l}
\dot{\mathbf{z}}=\mathbf{A}_{11} \mathbf{z}+\mathbf{A}_{12} \mathbf{w}, \\
\dot{\mathbf{w}}=\mathbf{A}_{21} \mathbf{z}+\mathbf{A}_{22} \mathbf{w}
\end{array}\right.
$$

where the matrices A's are of proper sizes. The exact MZ dynamics for the observed variable $\mathbf{z}$ is known as

$$
\frac{d \mathbf{z}}{d t}=\mathbf{A}_{11} \mathbf{z}+\mathbf{A}_{12} \int_{0}^{t} e^{\mathbf{A}_{22}(t-s)} \mathbf{A}_{21} \mathbf{z}(s) d s+\mathbf{A}_{21} e^{\mathbf{A}_{22} t} \mathbf{w}(0) .
$$

In our example, we set the domain of interest to be $D_{\mathbf{x}}=[-2,2]^{2}$. For data generation, we set all trajectory length to be $\left(n_{M}+2\right)$. Hence, each trajectory contributes to one data entry $\left(J_{0}=1\right)$ in the training data set [Eq. (20)]. Based on the solution behavior, two cases are presented here: (1) fast-decay case for $\alpha=2$; and (2) slow-decay case for $\alpha=1.1$.

We first consider the fast-decay case. In Fig. 2, we plot the NN model prediction of the observed variable $x_{1}$ for up to $t=20$, using memory term $n_{M}=30$, which corresponds to memory length $T_{M}=n_{M} \Delta=0.6$. We observe that, for four arbitrarily chosen initial conditions, the network predictions match the exact true solution well. Since the solution decays to zero fast, we did not conduct model prediction over the longer term.

Longer-term predictions are conducted for the slow-decay case with $\alpha=1.1$, as shown in Fig. 3. The NN model is constructed using memory lenght $T_{M}=0.6$. The model prediction again shows good agreement with the exact solution for up to $t=100$.

We then examine the effect of different memory length in the NN modeling. The prediction errors produced by the models with varying $n_{M}$ are shown in Fig. 4 . We observe that the NN models become more accurate as the memory length increases. The accuracy starts to saturate around $n_{M}=30$, which corresponds to memeory length $T_{M}=0.6$. Further increasing the memory length induces no further accuracy improvement. 


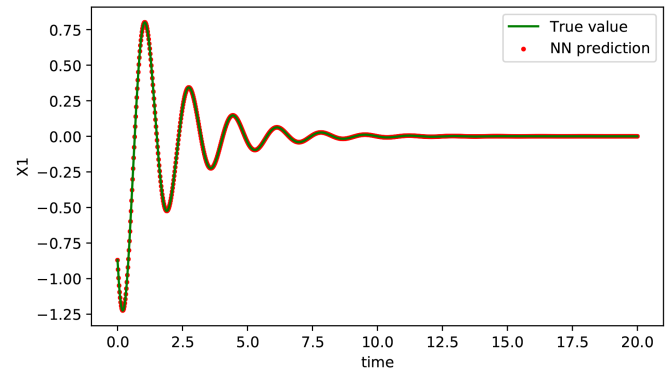

(a) Initial value $=(-0.87,0.65)$

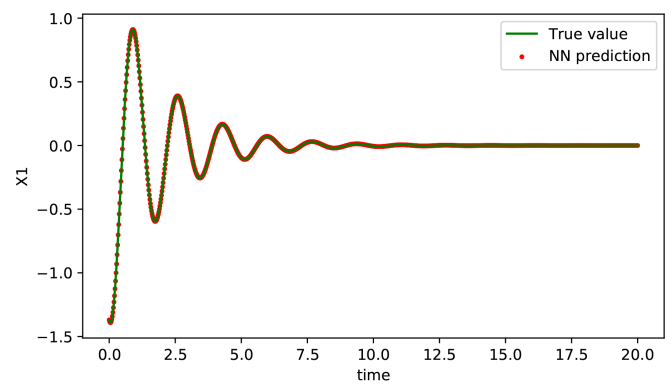

(c) Initial value $=(-1.37,-0.11)$

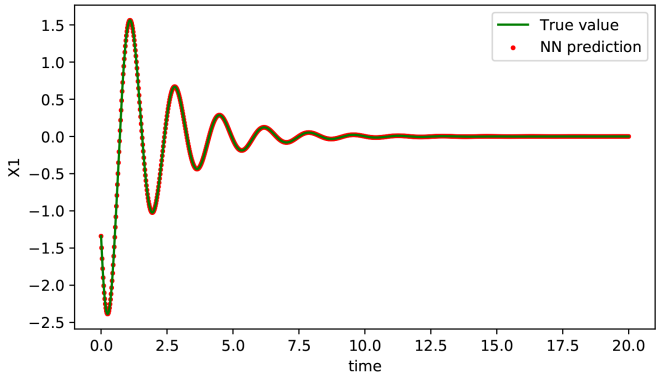

(b) Initial value $=(-1.34,1.7)$

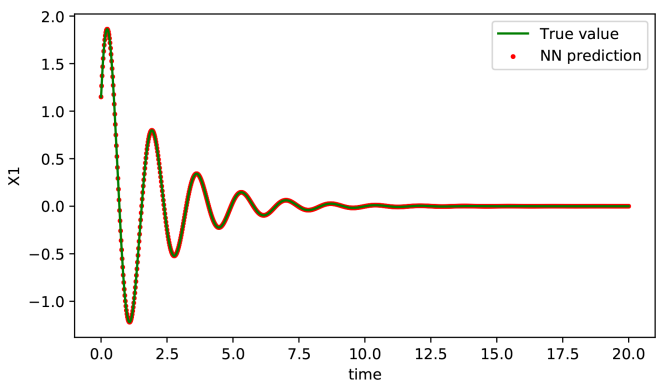

(d) Initial value $=(1.15,-1.21)$

FIG. 2: Example 1: Fast-decay case; neural network model prediction of $x_{1}$ with memory length $T_{M}=0.6$ using four different initial conditions

\subsection{Example 2: Nonlinear System}

We now consider a damped pendulum system, which is a simple nonlinear system.

$$
\left\{\begin{array}{l}
\dot{x}_{1}=x_{2}, \\
\dot{x}_{2}=-\alpha x_{2}-\beta \sin x_{1},
\end{array}\right.
$$

where $\alpha=0.1$ and $\beta=8.91$. The domain of interest is set as $D_{\mathbf{x}}=[-2,2] \times[-4,4]$. The observed variable is $z=x_{1}$ and we seek to construct an NN model for its prediction. Our training data sets [Eq. (20)] are generated by collecting $J_{0}=5$ sequences of $x_{1}$ data randomly from each $x_{1}$ trajectory data of length $K=50$. The memory step is tested for $n_{M}=3,5,8,10$, $13,15,18$, and 20 . The numerical errors in the model predictions with different memory steps are shown in Fig. 5. We observe that the accuracy improvement over increasing $n_{M}$ starts to saturate with $n_{M} \geq 10$. The NN model predictive result with $n_{M}=20$ is shown in Fig. 6. This corresponds to memory length $T_{M}=n_{M} \times \Delta=0.4$. We again observe very good agreement with the reference solution for long-term integration up to $t=100$.

\subsection{Example 3: Chaotic System}

We now consider a nonlinear chaotic system (Pavliotis and Stuart, 2008):

Volume 1, Issue 2, 2020 


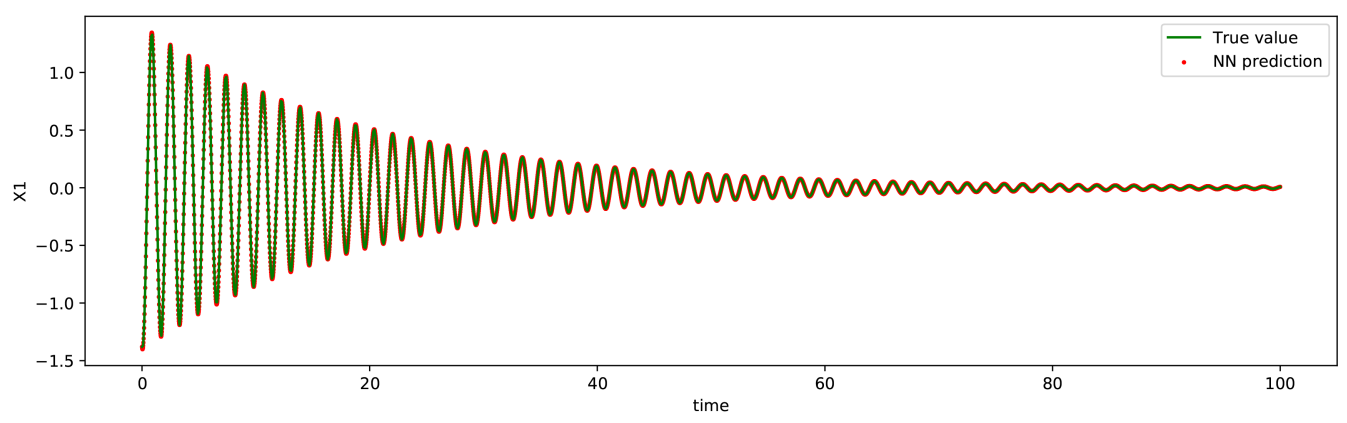

(a) Initial value $=(-1.38,-0.11)$

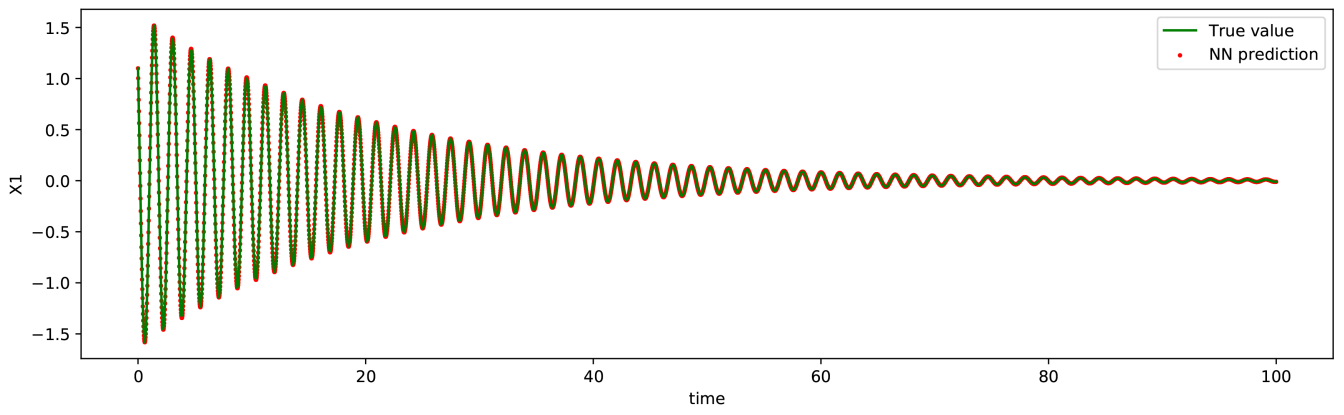

(b) Initial value $=(1.1,1.45)$

FIG. 3: Example 1: Slow-decay cases; neural network model prediction of $x_{1}$ with memory length $T_{M}=0.6$ using two different initial conditions

$$
\left\{\begin{array}{l}
\dot{x}_{1}=-x_{2}-x_{3}, \\
\dot{x}_{2}=x_{1}+\frac{1}{5} x_{2}, \\
\dot{x}_{3}=\frac{1}{5}+y-5 x_{3}, \\
\dot{y}=-\frac{y}{\epsilon}+\frac{x_{1} x_{2}}{\epsilon},
\end{array}\right.
$$

where $\epsilon>0$ is a small parameter. In this example, we choose the observed variables to be $\mathbf{z}=\left(x_{1}, x_{2}, x_{3}\right)^{\top}$ and let the fast variable $y$ be the unobserved variable. Note that for this system, there exists a homogenized system for the slow variables $\left(x_{1}, x_{2}, x_{3}\right)$,

$$
\left\{\begin{array}{l}
\dot{x}_{1}=-x_{2}-x_{3}, \\
\dot{x}_{2}=x_{1}+\frac{1}{5} x_{2}, \\
\dot{x}_{3}=\frac{1}{5}+x_{3}\left(x_{1}-5\right) .
\end{array}\right.
$$

The reduced system is a good approximation for the true system when $\epsilon \ll 1$. Here we will construct NN models for the reduced variables $\mathbf{z}$ and compare the prediction results against the true solution of Eq. (29), as well as those obtained by the reduced system [Eq. (30)]. We set $\epsilon=0.01$, in which case the reduced system [Eq. (30)] is considered an accurate approximation of the true system. 


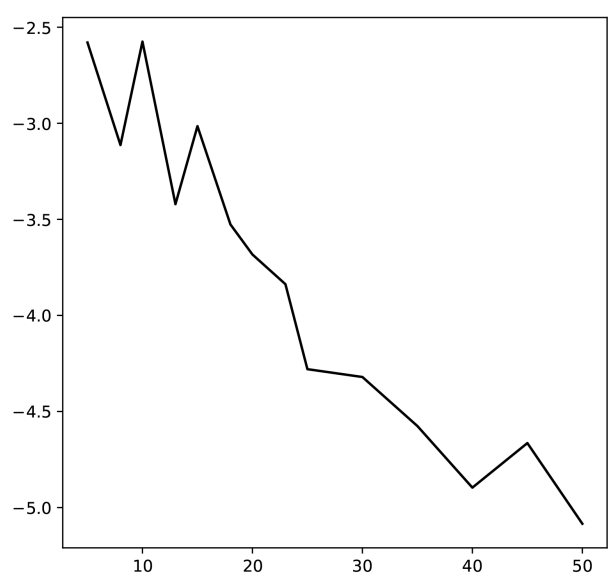

(a) Fast-decay case

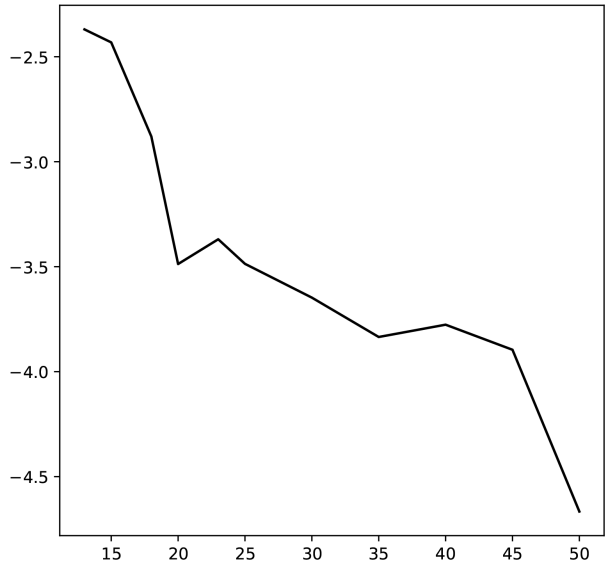

(b) Slow-decay case

FIG. 4: Example 1: NN prediction errors in log scale vs. number of memory terms

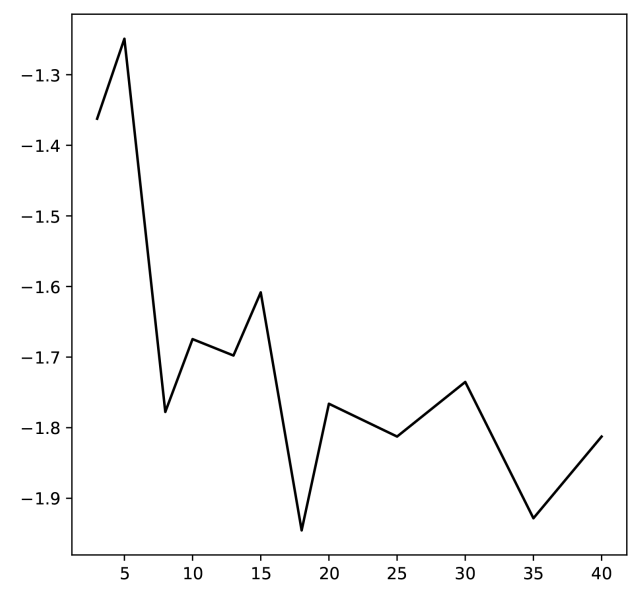

FIG. 5: Example 2: Prediction errors in log scale vs. the number of memory steps

The domain of interest is set to be $D_{\mathbf{x}}=[-7.5,10] \times[-10,7.5] \times[0,18] \times[-1,100]$, which is sufficiently large to enclose the solution trajectories for different initial conditions. To generate training data set [Eq. (20)], we solve the true system [Eq. (29)] using randomly sampled initial conditions via a high resolution numerical solver and record the trajectories of $\mathbf{z}$ with length $K=100$, which corresponds to a time lapse of $K \Delta=2$. In each trajectory, we randomly select $J_{0}=5$ sequences of data with length $\left(n_{M}+2\right)$ for our training data set. Different memory steps of $n_{M}=10,20,30,40,50,60,70$, and 80 are examined. This corresponds to memory length $T$ ranging from 0.2 to 1.6. Our results indicate that $n_{M}=60$ (i.e., $T_{M}=1.2$ ) delivers accurate predictions. Further increasing memory length does not lead to better predictions.

The evolution of prediction errors, measured in $\ell_{2}$-norm against the reference true solution, are shown in Fig. 7, for both our NN model and the reduced model [Eq. (30)] for long-term prediction up to $t=400$. Here the errors are averaged over 100 simulations using randomly selected initial conditions. We observe that our NN model produces noticebaly more accurate results than

Volume 1, Issue 2, 2020 


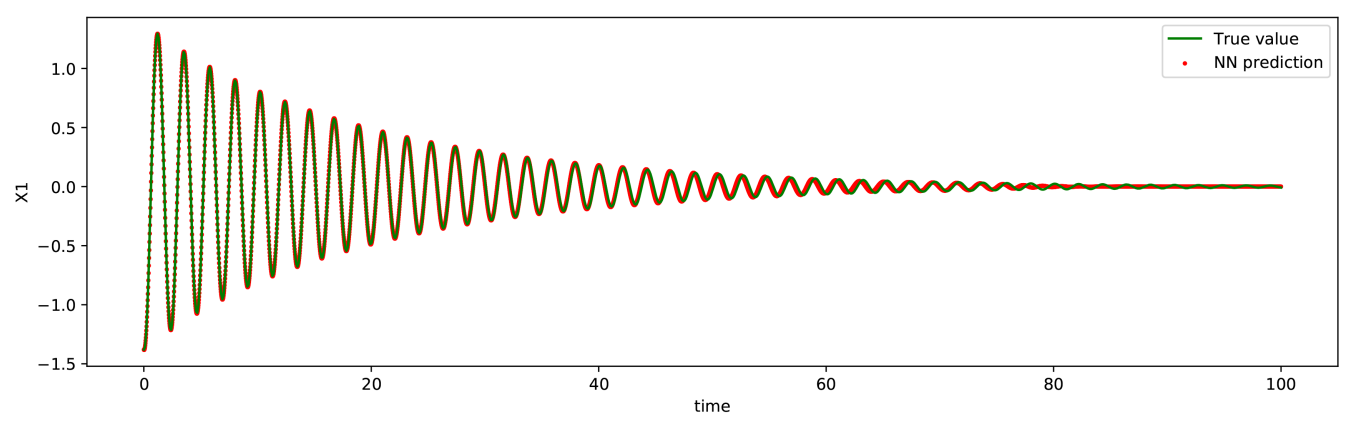

(a) Initial value $=(-1.38,-0.22)$

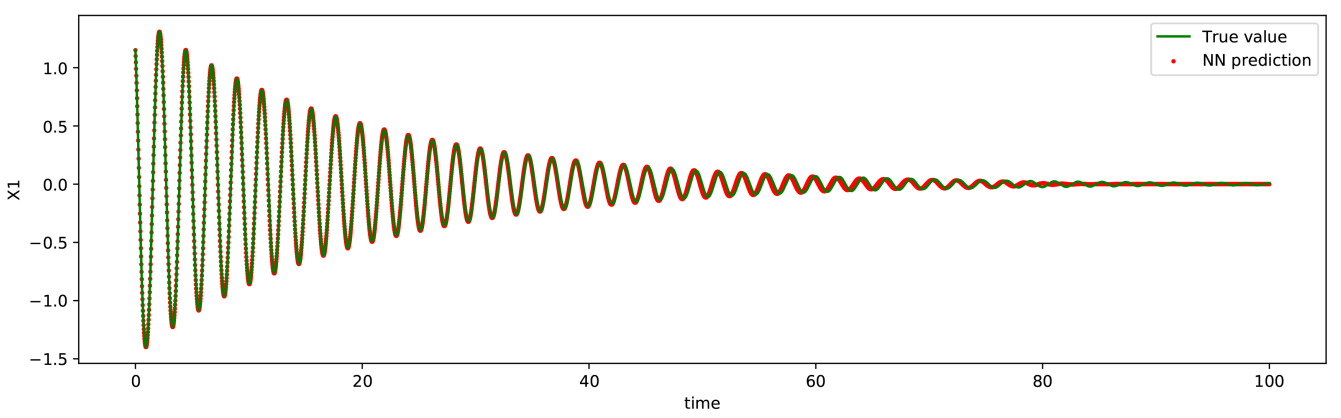

(b) Initial value $=(1.15,-2.43)$

FIG. 6: Example 2: NN model prediction of $x_{1}$ with memory length $T_{M}=0.4$ using two arbitrary initial conditions

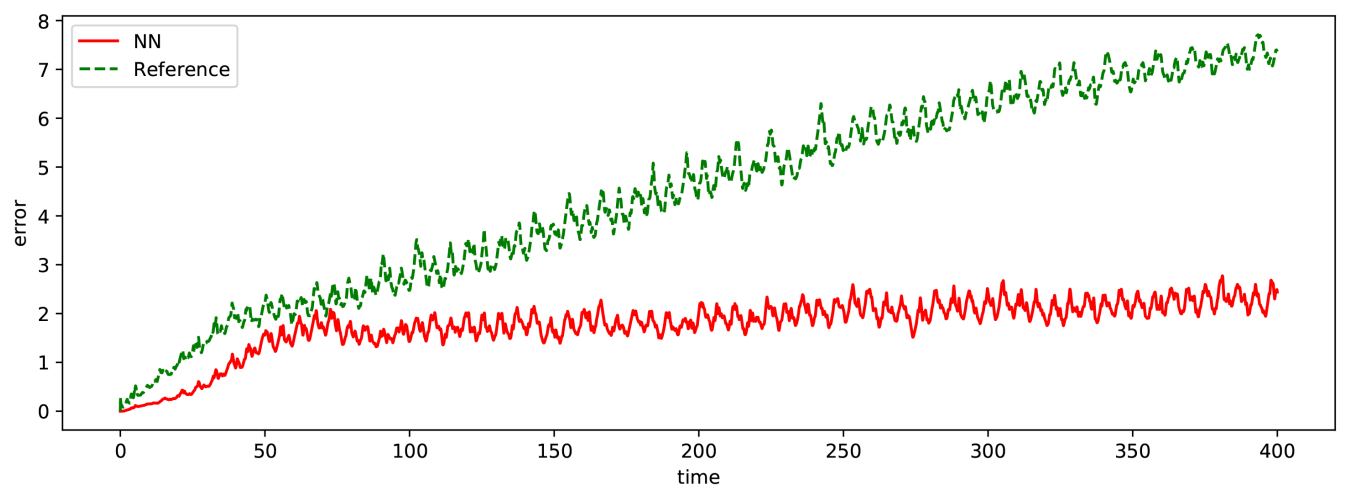

FIG. 7: Example 3: Errors in model predictions over time, by the NN model with $T_{M}=1.2$ and the reduced system [Eq. (30)]. The errors are averaged over 100 simulations of different initial conditions.

the reduced system [Eq. (30)], even for the case of small $\epsilon$ when Eq. (30) is supposed to be highly accurate. More importantly, the NN model exhibits much smaller error growth over a long time compared to the reduced system [Eq. (30)]. The solution behavior for long-term integration up to $t=400$ for the first component $x_{1}$ is shown in Fig. 8. (Behavior for other components is similar.) The NN model produces visually better results than the reduced system [Eq. (30)] when compared to the true solution, especially in term of capturing the phase/frequency of the solution. 


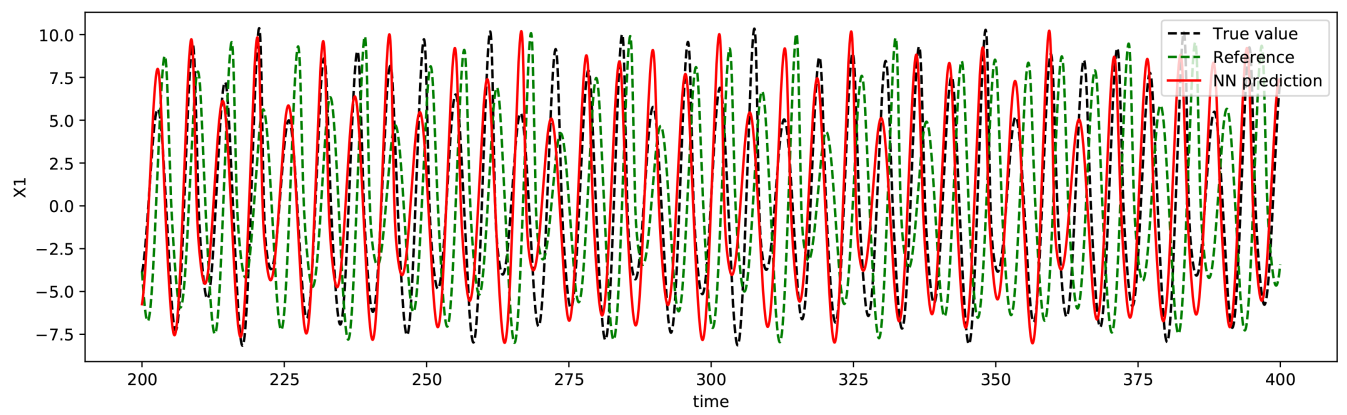

(a)

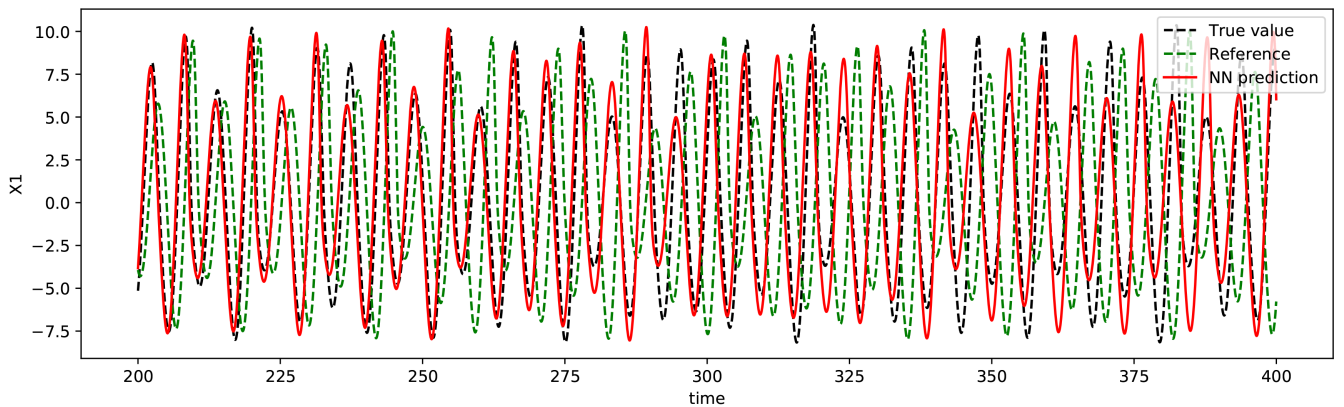

(b)

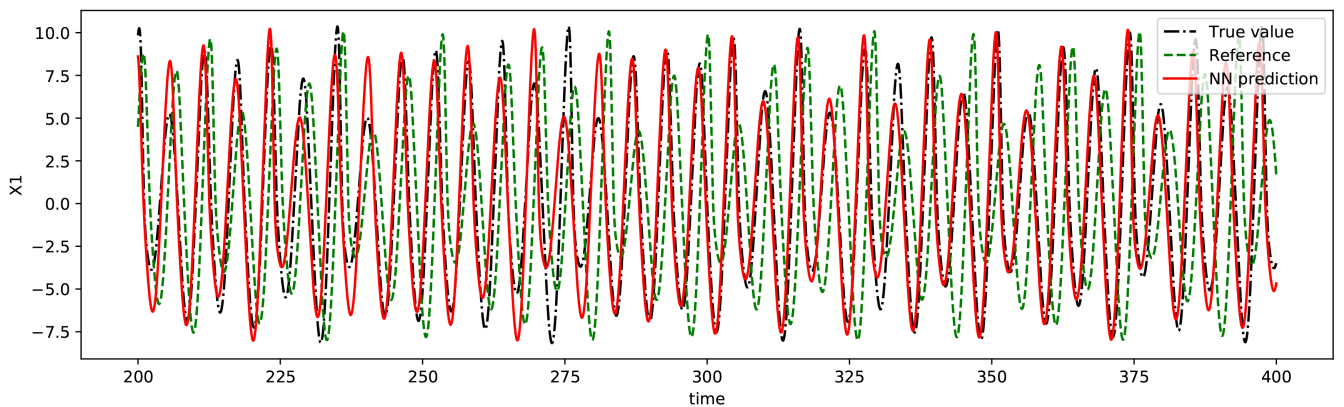

(c)

FIG. 8: Example 3: Long-term model prediction of $x_{1}$ by the NN model with $T_{M}=1.2$ and the reduced system [Eq. (30)]. Zoomed view for $t \in[200,400]$ with three aribitrarily chosen initial conditions.

\subsection{Example 4: Larger Linear System}

We now consider a larger linear system involving 20 state variables $\mathbf{x}=(\mathbf{p} ; \mathbf{q}) \in \mathbb{R}^{20}$,

$$
\left\{\begin{array}{l}
\dot{\mathbf{p}}=\Sigma_{11} \mathbf{p}+\left(\mathbf{I}+\Sigma_{12}\right) \mathbf{q} \\
\dot{\mathbf{q}}=-\left(\mathbf{I}+\Sigma_{21}\right) \mathbf{p}-\Sigma_{22} \mathbf{q}
\end{array}\right.
$$

where $\mathbf{p}, \mathbf{q} \in \mathbb{R}^{10}, \mathbf{I}$ is identity matrix of size $10 \times 10$, and $\Sigma_{i j} \in \mathbb{R}^{10 \times 10}, i=1,2, j=1,2$. 
We set the observed variables to be $\mathbf{z}=\mathbf{p} \in \mathbb{R}^{10}$ and let $\mathbf{w}=\mathbf{q} \in \mathbb{R}^{10}$ be the unobserved variables. Note that as a linear system [Eq. (26)], the exact MZ equations for the reduced variables $\mathbf{z}$ are available in analytical form Eq. (27). We set the entries of $\Sigma_{i j}$ to be small and consider them as perturbations to an oscillatory system. The exact values of the entries of matrices $\Sigma_{i j}$ are presented in the Appendix A, for completeness of the paper.

The domain of interest is set to be $D_{\mathbf{x}}=[-2,2]^{20}$. In generating the training data sets, we randomly select $J_{0}=5$ sequences of data from each trajectory of $\mathbf{z}$ with length $K=100$. We test different memory steps for $n_{M}=10,15,20,25,30,35,40,45$, and 50. Our experiments show that $n_{M}=30$, which corresponds to a memory length $T_{M}=0.6$, provides accurate prediction. Further increasing memory length does not lead to better prediction accuracy. Our $\mathrm{NN}$ model predictions for long-term integration up to $t=150$ are shown in Fig. 9. Compared to the reference solution obtained from the true system, we observe very good agreement, where the NN predictions overlap the true solutions to be visually indistinguishable.

\section{CONCLUSION}

We present construction of DNN models to approximating unknown dynamical systems when only a subset of variables are observed. The DNN model then provides a reduced model for the unknown dynamical system. Based on MZ formulation for reduced systems, we established a discrete MZ formulation with finite memory assumption. We then designed a straightforward DNN structure to explicitly incorporate the system memory into the predictive model. Numerical tests on both linear and nonlinear systems demonstrated good accuracy of the DNN models. This invites further in-depth study of the approach, both theoretically and numerically.

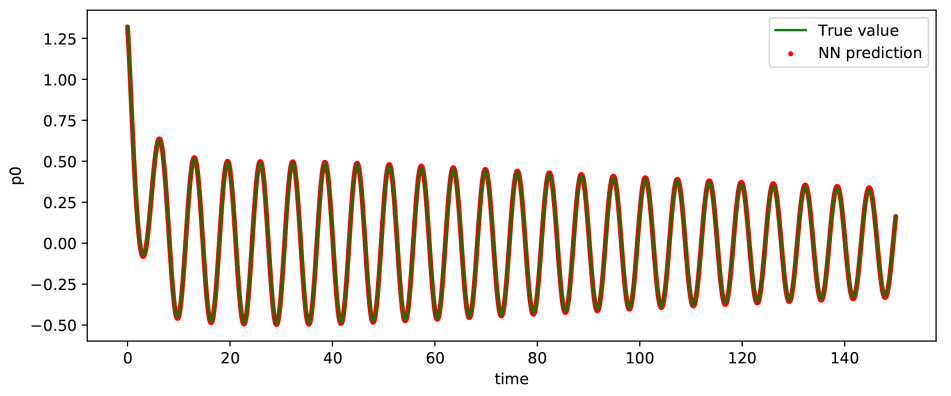

(a)

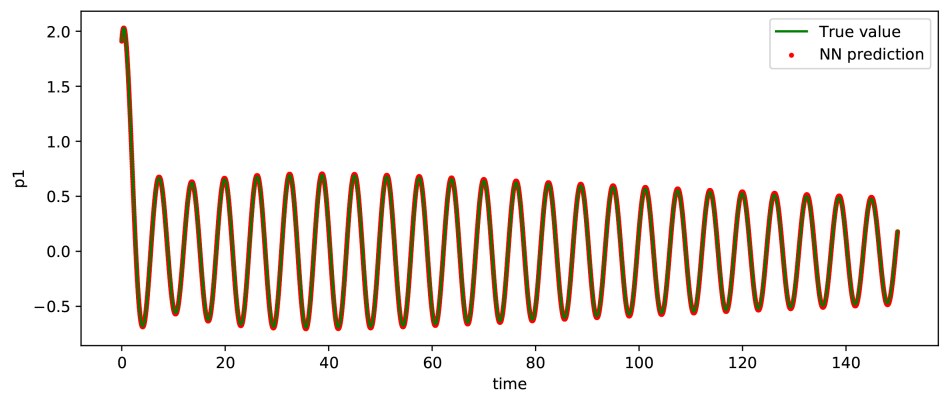

(b)

FIG. 9. 


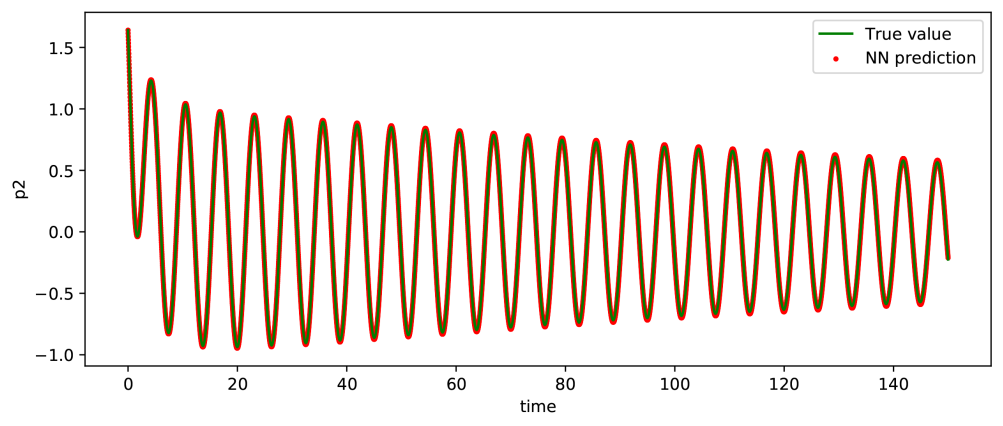

(c)

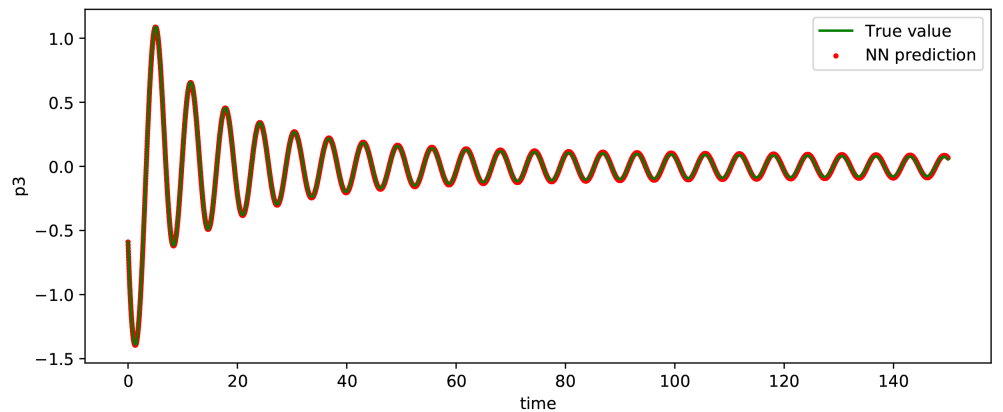

(d)

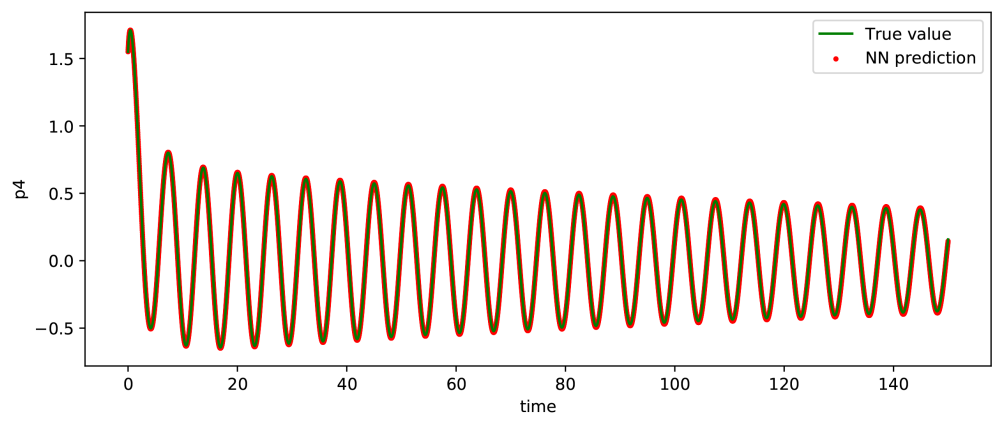

(e)

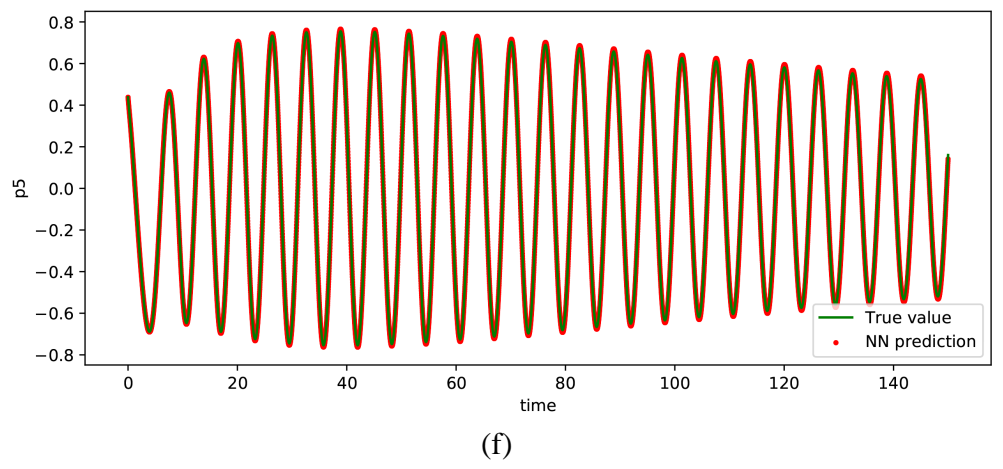

FIG. 9.

Volume 1, Issue 2, 2020 


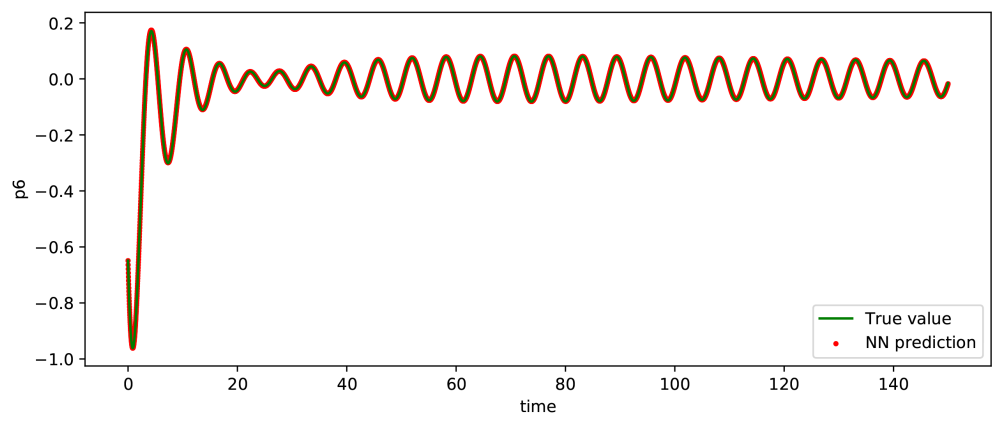

(g)

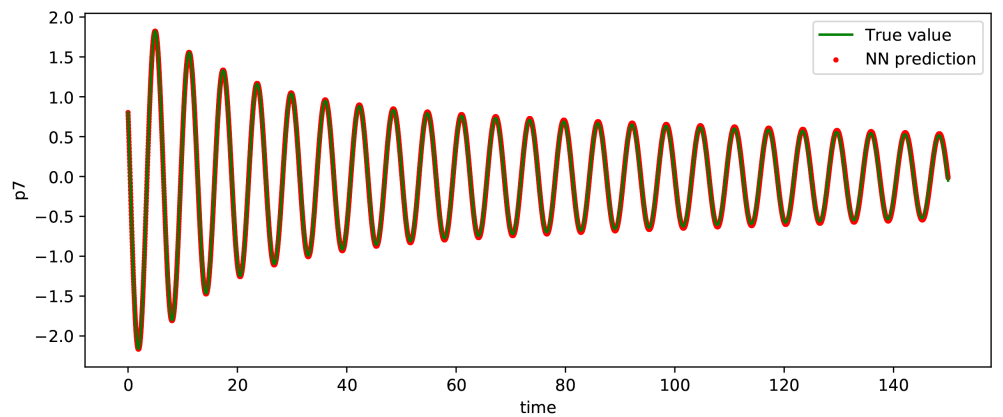

(h)

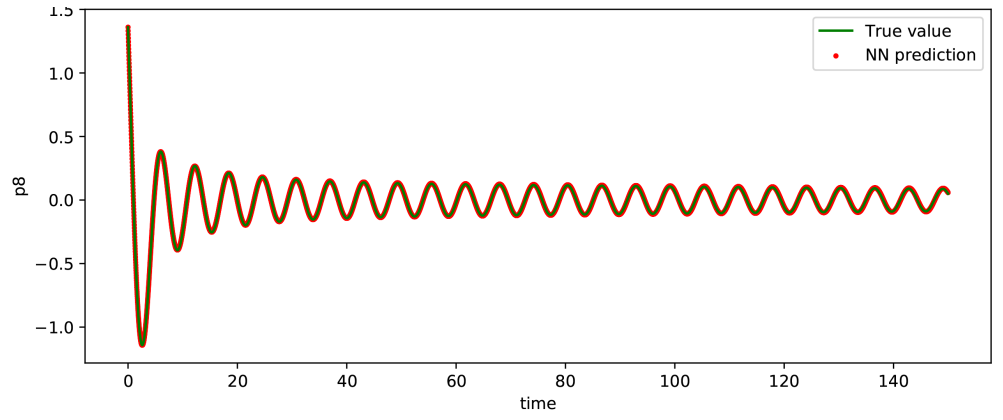

(i)

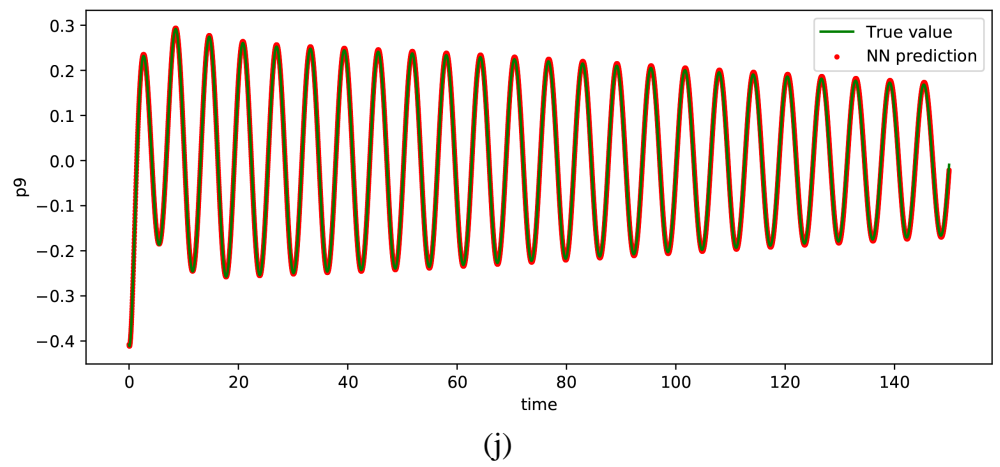

FIG. 9: Example 4: NN model prediction of $\mathbf{p}$ for up to $t=150$, with memory length $T_{M}=0.6$ 


\section{ACKNOWLEDGMENT}

This work was partially supported by AFOSR FA9550-18-1-0102.

\section{REFERENCES}

Bernstein, D., Optimal Prediction of Burgerss Equation, Multiscale Model. Simul., vol. 6, pp. 27-52, 2007.

Brennan, C. and Venturi, D., Data-Driven Closures for Stochastic Dynamical Systems, J. Comput. Phys., vol. 372, pp. 281-298, 2018.

Brunton, S.L., Proctor, J.L., and Kutz, J.N., Discovering Governing Equations from Data by Sparse Identification of Nonlinear Dynamical Systems, Proc. Natl. Acad. Sci., vol. 113, no. 15, pp. 3932-3937, 2016.

Chen, Z. and Xiu, D., On Generalized Residue Network for Deep Learning of Unknown Dynamical Systems, J. Comput. Phys., submitted, 2020.

Chertock, A., Gottlieb, D., and Solomonoff, A., Modified Optimal Prediction and Its Application to a Particle-Method Problem, J. Sci. Comput., vol. 37, no. 2, pp. 189-201, 2008.

Chorin, A.J., Hald, O.H., and Kupferman, R., Optimal Prediction with Memory, Physica D: Nonlinear Phenomena, vol. 166, nos. 3-4, pp. 239-257, 2002.

Hald, O. and Stinis, P., Optimal Prediction and the Rate of Decay for Solutions of the Euler Equations in Two and Three Dimensions, Proc. Natl. Acad. Sci., vol. 104, pp. 6527-6532, 2007.

He, K., Zhang, X., Ren, S., and Sun, J., Deep Residual Learning for Image Recognition, Proceedings of the IEEE Conference on Computer Vision and Pattern Recognition, pp. 770-778, 2016.

Kang, S.H., Liao, W., and Liu, Y., IDENT: Identifying Differential Equations with Numerical Time Evolution, 2019. arXiv: 1904.03538

Lei, H., Baker, N., and Li, X., Data-Driven Parameterization of the Generalized Langevin Equation, Proc. Natl. Acad. Sci., vol. 113, no. 50, pp. 14183-14188, 2016.

Long, Z., Lu, Y., and Dong, B., PDE-Net 2.0: Learning PDEs from Data with Numeric-Symbolic Hybrid Deep Network, 2018a. arXiv: 1812.04426

Long, Z., Lu, Y., Ma, X., and Dong, B., PDE-Net: Learning PDEs from Data, Proceedings of the 35th International Conference on Machine Learning, Proceedings of Machine Learning Research, J. Dy and A. Krause, Eds., vol. 80, Stockholm, Sweden: PMLR, pp. 3208-3216, 2018 b.

Mori, H., Transport, Collective Motion, and Brownian Motion, Progress Theor. Phys., vol. 33, no. 3, pp. 423-455, 1965

Pavliotis, G. and Stuart, A., Multiscale Methods: Averaging and Homogenization, Berlin: Springer, 2008.

Qin, T., Chen, Z., Jakeman, J., and Xiu, D., A Neural Network Approach for Uncertainty Quantification for Time-Dependent Problems with Random Parameters, Int. J. Uncertainty Quantif., submitted, 2020 .

Qin, T., Wu, K., and Xiu, D., Data Driven Governing Equations Approximation Using Deep Neural Networks, J. Comput. Phys., vol. 395, pp. 620-635, 2019a.

Qin, T., Wu, K., and Xiu, D., Structure-Preserving Method for Reconstructing Unknown Hamiltonian Systems from Trajectory Data, SIAM J. Sci. Comput., submitted, $2019 \mathrm{~b}$.

Raissi, M., Deep Hidden Physics Models: Deep Learning of Nonlinear Partial Differential Equations, $J$. Mach. Learn. Res., vol. 19, no. 25, pp. 1-24, 2018.

Raissi, M., Perdikaris, P., and Karniadakis, G.E., Physics Informed Deep Learning (Part I): Data-Driven Solutions of Nonlinear Partial Differential Equations, 2017a. arXiv: 1711.10561

Volume 1, Issue 2, 2020 
Raissi, M., Perdikaris, P., and Karniadakis, G.E., Physics Informed Deep Learning (Part II): Data-Driven Discovery of Nonlinear Partial Differential Equations, 2017b. arXiv: 1711.10566

Raissi, M., Perdikaris, P., and Karniadakis, G.E., Multistep Neural Networks for Data-Driven Discovery of Nonlinear Dynamical Systems, 2018. arXiv: 1801.01236

Rudy, S.H., Brunton, S.L., Proctor, J.L., and Kutz, J.N., Data-Driven Discovery of Partial Differential Equations, Sci. Adv., vol. 3, no. 4, p. e1602614, 2017.

Rudy, S.H., Kutz, J.N., and Brunton, S.L., Deep Learning of Dynamics and Signal-Noise Decomposition with Time-Stepping Constraints, J. Comput. Phys., vol. 396, pp. 483-506, 2019.

Schaeffer, H., Learning Partial Differential Equations via Data Discovery and Sparse Optimization, Proc. Royal Soc. London A: Math. Phys. Eng. Sci., vol. 473, no. 2197, 2017.

Schaeffer, H. and McCalla, S.G., Sparse Model Selection via Integral Terms, Phys. Rev. E, vol. 96, no. 2, p. 023302, 2017.

Schaeffer, H., Tran, G., and Ward, R., Extracting Sparse High-Dimensional Dynamics from Limited Data, SIAM J. Appl. Math., vol. 78, no. 6, pp. 3279-3295, 2018.

Stinis, P., Higher Order MoriZwanzig Models for the Euler Equations, Multiscale Model. Simul., vol. 6, pp. 741-760, 2007.

Sun, Y., Zhang, L., and Schaeffer, H., NEUPDE: Neural Network based Ordinary and Partial Differential Equations for Modeling Time-Dependent Data, 2019. arXiv: 1908.03190

Tibshirani, R., Regression Shrinkage and Selection via the Lasso, J. R. Stat. Soc. B (Methodological), pp. 267-288, 1996.

Tran, G. and Ward, R., Exact Recovery of Chaotic Systems from Highly Corrupted Data, Multiscale Model. Simul., vol. 15, no. 3, pp. 1108-1129, 2017.

Venturi, D. and Karniadakis, G., Convolutionless Nakajima-Zwanzig Equations for Stochastic Analysis in Nonlinear Dynamical Systems, Proc. R. Soc. A, vol. 470, no. 2166, p. $20130754,2014$.

Wang, Q., Ripamonti, N., and Hesthaven, J., Recurrent Neural Network Closure of Parametric PODGalerkin Reduced-Order Models based on the Mori-Zwanzig Formalism, J. Comput. Phys., vol. 410, 2020.

Wu, K., Qin, T., and Xiu, D., Structure-Preserving Method for Reconstructing Unknown Hamiltonian Systems from Trajectory Data, 2019. arXiv: 1905.10396

Wu, K. and Xiu, D., Numerical Aspects for Approximating Governing Equations Using Data, J. Comput. Phys., vol. 384, pp. 200-221, 2019.

Wu, K. and Xiu, D., Data-Driven Deep Learning of Partial Differential Equations in Modal Space, J. Comput. Phys., vol. 408, p. 109307, 2020.

Zhu, Y. and Venturi, D., Faber Approximation of the Mori-Zwanzig Equation, J. Comput. Phys., vol. 372, pp. 694-718, 2018.

Zwanzig, R., Nonlinear Generalized Langevin Equations, J. Stat. Phys., vol. 9, no. 3, pp. 215-220, 1973.

\section{APPENDIX A. DETAILS OF EXAMPLE 4}

The detailed setting of Example 4 is

$$
\left\{\begin{array}{l}
\dot{\mathbf{p}}=\Sigma_{11} \mathbf{p}+\left(\mathbf{I}+\Sigma_{12}\right) \mathbf{q} \\
\dot{\mathbf{q}}=-\left(\mathbf{I}+\Sigma_{21}\right) \mathbf{p}-\Sigma_{22} \mathbf{q}
\end{array}\right.
$$


where $\mathbf{p}, \mathbf{q} \in \mathbb{R}^{10}, \mathbf{I}$ is identity matrix of size $10 \times 10$, and $\Sigma_{i j} \in \mathbb{R}^{10 \times 10}, i=1,2, j=1,2$. The matices $\Sigma_{i j}$ are defined as follows.

$$
\begin{gathered}
\Sigma_{11} \times 10^{3}= \\
\left.\begin{array}{rrrrrrrrrr}
-6.09 & 5.79 & -0.945 & -12.1 & 9.38 & -12.4 & 4.92 & 3.71 & 1.17 & 4.73 \\
-9.57 & -8.88 & -12.1 & -12.9 & 5.11 & 26.5 & -7.33 & -8.01 & -21.6 & -10.2 \\
-0.733 & 6.2 & 10.7 & -6.06 & -7.07 & -1.7 & -16.4 & 6.69 & -1.59 & 7.69 \\
7.83 & 12.5 & 5.77 & -14.9 & -17.8 & -1.01 & -4.05 & -15 & -6.61 & -4.94 \\
8.1 & 4.13 & 4.21 & 23.3 & -4.63 & 1.77 & -14.9 & 17.9 & -17.1 & -8.19 \\
-7.68 & 6.98 & 27.6 & 19 & 20.9 & 12.2 & 15.6 & -11.2 & -3.56 & -2.47 \\
-14.9 & -5.73 & -19.7 & -8.77 & -9.17 & -2.95 & -9.48 & -2.95 & 5.43 & 15.4 \\
-1.84 & 2.05 & -1.98 & 3.83 & -4.06 & 7.72 & 4.04 & -13.7 & 20.3 & 0.509 \\
12.1 & 19.7 & -14.3 & 12.6 & -4.67 & 9.72 & 5.87 & 0.664 & -10.8 & -18.2 \\
3.07 & 3.65 & 3.88 & 7.44 & 12.7 & 13.5 & -6.66 & -23.9 & -11.7 & 16.6
\end{array}\right),
\end{gathered}
$$

$$
\begin{aligned}
& \Sigma_{12} \times 10^{3}= \\
& \left(\begin{array}{rrrrrrrrrr}
11.7 & -12.3 & -8.87 & -6.86 & -9.6 & 11 & 25.6 & -0.155 & 17.8 & -10.9 \\
12.9 & 3.28 & 2.84 & 3.35 & 16.6 & 5.96 & 6.99 & -20.2 & 8.37 & -8.87 \\
-0.154 & -16.5 & 12.1 & 0.381 & 11.2 & -2.59 & 12.8 & 3.32 & -10.9 & -3.81 \\
6.49 & 15.8 & -0.273 & 9.05 & -3.15 & 0.976 & -7.35 & 0.889 & 6.41 & 15.6 \\
4.86 & -1.52 & 0.118 & 17.8 & -5.08 & -4.96 & -2.89 & 3 & 22.4 & 16.4 \\
7.83 & -9.66 & -2.09 & 5.97 & 3.97 & 19.2 & 4.03 & -15.3 & -8.5 & -15.8 \\
-4.61 & -4.98 & 17 & -14 & -17.5 & 0.104 & -27.5 & 10.9 & -17.9 & -5.9 \\
3.88 & 14 & -2.63 & -7.27 & -21 & -0.403 & -2.18 & -22 & 2.01 & -2.45 \\
14.4 & -4.65 & -8.67 & -23.2 & -2.73 & 9.58 & -13.9 & 0.415 & 10.3 & 17.5 \\
-16.8 & 8.18 & -12.3 & 14.2 & -18.4 & -10.2 & -11.4 & -1.99 & -2.65 & -2.34
\end{array}\right),
\end{aligned}
$$

$$
\begin{aligned}
& \Sigma_{21} \times 10^{3}= \\
& \left(\begin{array}{rrrrrrrrrr}
-3.51 & -4.91 & -4.51 & -15.8 & -12 & -5.72 & -9.52 & -14.3 & 0.745 & -11.8 \\
1.8 & 2.07 & 8.78 & 5.3 & -5.25 & 5.7 & 0.0957 & 9.77 & 2.17 & 12.8 \\
-9.87 & 5.19 & 0.884 & 2.59 & -7.95 & 5.56 & 6.41 & 16.4 & 15.6 & 14.3 \\
10.4 & 7.14 & 15.5 & -6.6 & 5.33 & -3.37 & 2.8 & -9.61 & 8 & -16.8 \\
15.5 & 19.6 & -1.1 & 0.6 & 8.38 & 7.62 & 3.43 & 1.28 & 10.3 & -4.76 \\
0.119 & -9.43 & -6.6 & -9.99 & -10.5 & 17.8 & 13.5 & -6.63 & -0.566 & -1.81 \\
-6.77 & -1.42 & 7.46 & 3.32 & 11.7 & 1.3 & -6.21 & 6.9 & 3.89 & 18.9 \\
2.93 & 15.1 & -4.65 & 11.1 & 9.13 & -9.58 & -7.04 & 6.88 & -4.07 & 10.2 \\
-6.02 & 14 & -5.91 & -4.92 & 0.851 & 0.652 & -2.57 & 0.835 & -5.14 & 10.6 \\
1.41 & 5.8 & -2.31 & 6.17 & 13.3 & 3.57 & 15.9 & -0.753 & -0.818 & -10.3
\end{array}\right),
\end{aligned}
$$




$\Sigma_{22} \times 10^{3}=$
$\left(\begin{array}{rrrrrrrrrr}1500 & 124 & 814 & -104 & -179 & -223 & -731 & -189 & -400 & 242 \\ 124 & 836 & 679 & 277 & 197 & -515 & -52.1 & -273 & 101 & 301 \\ 814 & 679 & 1500 & 651 & 755 & -605 & -379 & -546 & -225 & 223 \\ -104 & 277 & 651 & 1960 & 720 & -782 & -299 & -775 & -180 & 506 \\ -179 & 197 & 755 & 720 & 2290 & -973 & 518 & -19.1 & -604 & -369 \\ -223 & -515 & -605 & -782 & -973 & 1290 & -400 & 412 & 314 & -420 \\ -731 & -52.1 & -379 & -299 & 518 & -400 & 1960 & 68.3 & 455 & -316 \\ -189 & -273 & -546 & -775 & -19.1 & 412 & 68.3 & 576 & -53.6 & -332 \\ -400 & 101 & -225 & -180 & -604 & 314 & 455 & -53.6 & 1030 & 265 \\ 242 & 301 & 223 & 506 & -369 & -420 & -316 & -332 & 265 & 1090\end{array}\right)$.

